\title{
Effect of a Brief Family-Based Treatment on Complicated Grief in Bereaved Older Adults
}

\author{
Ilsung Nam \\ Department of Social Welfare, Sungkonghoe University, Seoul, Korea
}

Corresponding Author:

Ilsung $\mathrm{Nam}, \mathrm{PhD}$

https://orcid.org/0000-0002-2395-8938

Department of Social Welfare,

Sungkonghoe University, 320

Yeondong-ro, Guro-gu, Seoul 08359,

Korea

Tel: $+82-2-2610-4109$

Fax: +82-2-2683-8858

E-mail: ilsungn@skhu.ac.kr

Received: February 23, 2018

Revised: March 14, 2018

Accepted: March 20, 2018
Background: The current study compared the effectiveness of family-based complicated grief therapy with individual-based complicated grief therapy on improvement in complicated grief symptoms of bereaved older adults. Methods: Two community senior centers referred 29 older adults who lost their spouses. The study used a single-blinded randomized trial design, comparing family-based complicated grief therapy with individual-based complicated grief therapy on complicated grief symptoms and social impairment. Results: Relative to individual-based complicated grief therapy, participants in the family-based complicated grief therapy group demonstrated improvement in social impairments and complicated grief symptoms. Conclusion: The findings suggest that a family-based delivery model for complicated grief may be superior to an individually provided approach in reducing complicated grief symptoms, and in improving work and social functions in bereavement. (Ann Geriatr Med Res 2018;22:94-97)

Key Words: Bereavement, Family research, Grief, Therapy

\section{INTRODUCTION}

Complicated grief is a persistent and intense type of grief that can lead to adverse health outcomes including sleep disturbances, high blood pressure, and suicidal ideation. ${ }^{1,2)}$ Debilitating impairment in practical areas of functioning, including social and occupational areas, makes complicated grief a particularly difficult problem for clinical practice. ${ }^{3)}$

There is growing evidence supporting the effectiveness of complicated grief therapy (CGT) for complicated grief. CGT is a psychotherapeutic treatment approach consisting of revisiting the loved one's loss and re-envisioning one's own future life without the deceased person. ${ }^{4)}$ Some trials of CGT have reported beneficial effects on complicated grief. However, in previous studies, treatment was delivered via either individual- or group-based forms, ${ }^{4-7)}$ making between-modality comparisons difficult. Thus, the effectiveness of CGT by treatment modality remains unclear, and it is critical to determine the most effective form of intervention modality and develop appropriate intervention components for the modality.

A family-based intervention, by harnessing families' inherent strengths and bolstering capacity to cope with the loss, could be an effective intervention model for grief symptoms. ${ }^{8}$ Thus, a family-based form of CGT may be effective in reducing complicated grief symptoms and improving functions in social and work life areas.
The current study compared the effectiveness of familybased CGT (FCGT) with individual-based CGT (ICGT) in reducing $\mathrm{CG}$ and functional impairment.

\section{MATERIALS AND METHODS}

This study employed a study flow chart (CONSORT or COPE), comparing FCGT with ICGT in 29 older adults who had recently lost a spouse and experienced significant impairments in psychological and social areas of functioning due to the loss. Individual were referred to our research team for participation by 2 community senior centers. These centers provide bereavement support programs to surviving family members who have lost their loved ones to death. Additional inclusion criteria were a score above 25 on the inventory of complicated grief ${ }^{9)}$ and at least six months of elapsed time since loss, ${ }^{3{ }^{3}}$ and therefore, the recommendation to receive professional care for complicated grief. Exclusion criteria were suicidal risk, severe substance dependence, and a history of psychosis.

Intervention procedures were approved by the Institutional Review Board of Hallym University (approval number: 2016010). After providing written consent and under the supervision of trained psychologists and/or social workers with $\mathrm{PhD}$ degrees, participants completed the screening assessments. The current study was designed to find a moderate effect size $(\mathrm{d}=0.5)$ in the differences between FCGT and ICGT on psychological measures of grief. 
Using a computer-generated program, the individuals were randomly assigned to FCGT or ICGT group. Fifteen participants were assigned to the FCGT group, and fourteen were assigned to the ICGT group.

CGT is a modified interpersonal psychotherapy designed to incorporate Bowlby's attachment model of grief and strategies that have been used to target trauma-related symptoms. A complete description of CGT has been provided elsewhere. ${ }^{4}$ Eight weekly 60-minute sessions were conducted. Thirteen individuals received ICGT, which is a brief version of CGT. ICGT comprises the following: (1) psychoeducation about complicated grief, (2) emotional management, (3) revisiting the story of the death, (4) an imaginary conversation with the deceased, (5) situation revisiting, and (6) self-care planning in bereavement. Griefmonitoring homework was assigned for each session, with a grief-monitoring diary, wherein participants daily recorded their most distressing moments. This diary enables the examination of patterns of distressing moments in an individual and allows for reviewing such patterns during the intervention sessions. The ICGT participants individually received (1) through (6) listed above. FCGT participants individually received (1) through (4). For these participants, (5) and (6) involved family-based consultations to foster family help to eliminate restrictions in activities following the death and plan elimination of ongoing practical problems.

Assessments were conducted pre- and postintervention. complicated grief was measured using the ICG, which consists of 19 pathologic forms of grief reactions that participants rate on a 5-point Likert scale. Functional impairment was measured via the Work and Social Adjustment Scale. The total scores on the 2 outcome measures were used for analysis, controlling for the confounding factors of age, sex, and time since loss. Raters were blinded to the intervention assignment. Three raters with $\mathrm{PhD}$ degrees in social work or psychology and with experience working with bereaved older adults were trained by the author using the manual. There were no statistically significant differences between the raters in their scoring of the outcome variables.

The effect sizes for the outcome measures were calculated by subtracting preintervention Cohen $\mathrm{d}$ from postintervention Cohen d. Analysis of covariances (ANCOVAs) were performed to examine the effects of intervention assignment on the outcome measures, adjusting for age, sex, and time since loss.

\section{RESULTS}

Demographic information on the 29 bereaved spouses is provided in Table 1. Fifteen of the participants were female and fourteen were male. The average age was 69.66 (standard deviation [SD], 13.13) years, and the average time since loss was 265.59 (SD, 20.24) days at the time of referral. Demographic information of the FCGT and the

Table 1. Demographic information of the study participants

\begin{tabular}{|c|c|c|c|}
\hline \multirow{2}{*}{ Variable } & \multirow{2}{*}{ Total } & \multicolumn{2}{|c|}{ Intervention assignment } \\
\hline & & FCGT & ICGT \\
\hline Sex & $29(100)$ & & \\
\hline Female & $14(48.28)$ & $8(53.33)$ & $6(42.86)$ \\
\hline Male & $15(51.62)$ & $7(46.67)$ & $8(57.14)$ \\
\hline Age (yr) & $69.66 \pm 13.13$ & $68.62 \pm 14.69$ & $70.38 \pm 13.01$ \\
\hline Time since loss (day) & $265.59 \pm 20.24$ & $267.32 \pm 23.22$ & $254.01 \pm 16.27$ \\
\hline
\end{tabular}

Values are presented as number (\%) or mean \pm standard deviation.

FCGT, family-based complicated grief therapy; ICGT, individual-based complicated grief therapy.
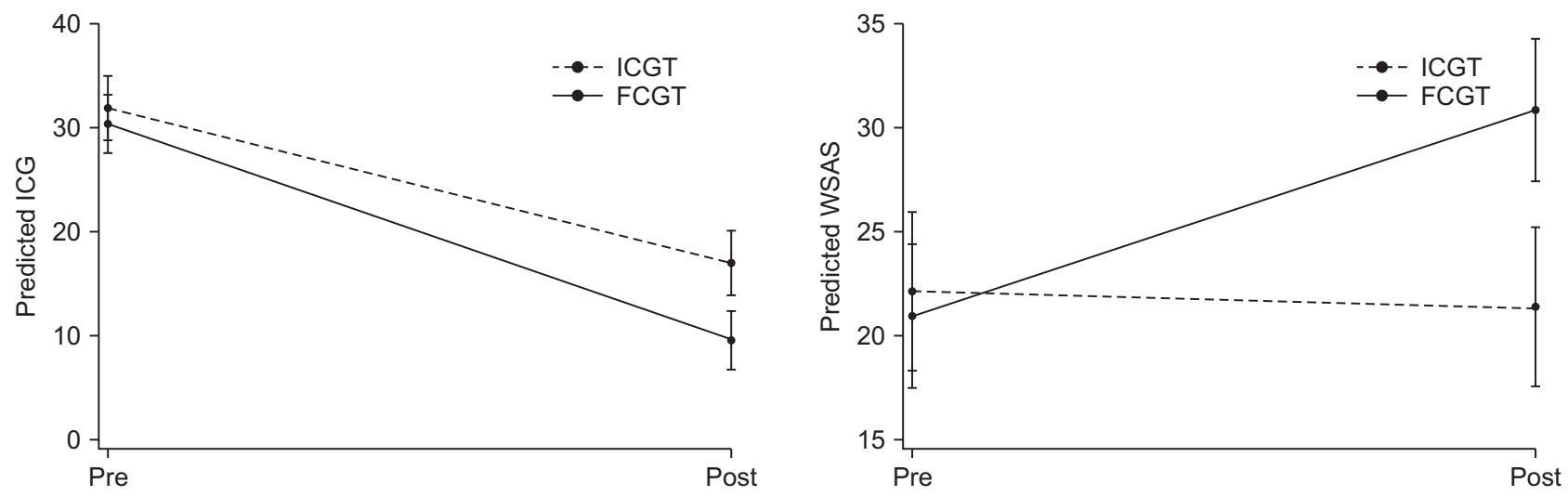

Fig. 1. Improvement in complicated grief and Work and Social Adjustment Scale (WSAS) scores as a function of family-based complicated grief therapy (FCGT) versus individual-based complicated grief therapy (ICGT). 
ICGT groups is presented in Table 1. There were no significant differences between the intervention groups in terms of sex, age, and time since loss. There were also no significant differences in preintervention complicated grief scores between FCGT and ICGT participants.

There was a significant improvement in complicated grief and Work and Social Adjustment Scale scores in FCGT versus ICGT (Fig. 1). The mean scores of complicated grief in FCGT were 30.38 (SD, 6.06) and 9.63 (SD, 2.92) at pre- and postintervention, respectively, whereas the mean scores of complicated grief in ICGT were 31.85 (SD, 7.60) and 17.00 (SD, 5.35) at pre- and postintervention, respectively. Cohen $\mathrm{d}$ for complicated grief scores was 1.16, indicating a large effect size. The mean scores of Work and Social Adjustment Scale in FCGT were 20.94 (SD, 7.99) and 30.81 (SD, 4.69) at pre- and postintervention, respectively, whereas the mean scores of Work and Social Adjustment Scale in ICGT were 22.15 (SD, 8.38) and 21.38 (SD, 5.85) at pre- and postintervention, respectively. Cohen $\mathrm{d}$ for complicated grief scores was -1.19 , indicating a large effect size.

The ANCOVA results indicated that FCGT was superior to ICGT with respect to complicated grief and Work and Social Adjustment Scale scores. There was a significant effect of intervention condition on postintervention complicated grief, adjusting for baseline ICG score, age, sex, and time since loss $(F[1]=9.05, p=0.004)$. There was also a significant effect of intervention condition on postintervention Work and Social Adjustment Scale, adjusting for baseline Work and Social Adjustment Scale score, age, sex, and time since loss $(\mathrm{F}[1]=5.14, \mathrm{p}=0.026)$. However, there were no interaction effects of intervention assignment with time $(\mathrm{p}=0.078)$.

\section{DISCUSSION}

The purpose of the current study was to compare FCGT with ICGT in reducing complicated grief, by using a randomized trial design. There were significant differences between FCGT and ICGT, which has critical implications for the development of grief interventions specifically and for future research generally. First, the complicated grief and Work and Social Adjustment Scale scores of the FCGT group significantly improved, relative to those of the ICGT group. It appears that family consultations on situational revisiting and self-care planning were effective in improving complicated grief and Work and Social Adjustment Scale scores. The promising effects of FCGT might be the result of family consultations preventing family-related dysfunction which is likely associated with avoidance of grief that leads to maladaptive grief reactions." Additionally, family consultations may have helped the bereaved family members to become actively involved in daily activities, such as housework, job, and financial tasks associated with the death, as indicated by the large effect size in Work and Social Adjustment Scale scores.
Although the present study has important clinical implications for the treatment of complicated grief, it is limited because changes in family function during the study period could not be examined. Future research should examine whether FCGT has beneficial effects on family function and thereby on complicated grief. This will help in understanding the mechanism by which family-based intervention is superior to individually provided intervention in reducing complicated grief. Another limitation is that the current study did not examine other important variables that might affect complicated grief in individuals bereaved by an accident. For example, posttraumatic symptoms may be a confounder in predicting complicated grief because of the trauma associated with bereavement due to an accident. Replication with a larger sample is warranted to support the conclusions of the present study.

In conclusion, the findings that FCGT helped improve social impairments and complicated grief symptoms, relative to ICGT, provide important insights into the development of CGT, which may enhance the effectiveness of therapeutic interventions for complicated grief in conjugally bereaved older adults. Understanding the mechanisms of the relationship between the family-based intervention modality and complicated grief is also critical for developing effective interventions for bereaved individuals.

Conflicts of Interest Disclosures: The researchers claim no conflicts of interest.

\section{ACKNOWLEDGMENTS}

This work was supported by the National Research Foundation of Korea Grant funded by the Korean Government (NRF-2012S1A6A3A01033504).

\section{REFERENCES}

1. Germain A, Caroff K, Buysse DJ, Shear MK. Sleep quality in complicated grief. J Trauma Stress 2005;18:343-6.

2. Prigerson HG, Bierhals AJ, Kasl SV, Reynolds CF 3rd, Shear MK, Day N, et al. Traumatic grief as a risk factor for mental and physical morbidity. Am J Psychiatry 1997;154:616-23.

3. Prigerson HG, Horowitz MJ, Jacobs SC, Parkes CM, Aslan M, Goodkin K, et al. Prolonged grief disorder: psychometric validation of criteria proposed for DSM-V and ICD-11. PLoS Med 2009;6: e1000121.

4. Shear K, Frank E, Houck PR, Reynolds CF 3rd. Treatment of complicated grief: a randomized controlled trial. JAMA 2005;293: 2601-8.

5. Shear MK, Wang Y, Skritskaya N, Duan N, Mauro C, Ghesquiere A. Treatment of complicated grief in elderly persons: a randomized clinical trial. JAMA Psychiatry 2014;71:1287-95.

6. Supiano KP, Luptak M. Complicated grief in older adults: a randomized controlled trial of complicated grief group therapy. Gerontologist 2014;54:840-56. 
7. Wagner B, Maercker A. A 1.5-year follow-up of an Internet-based intervention for complicated grief. J Trauma Stress 2007;20:6259.

8. Kissane DW, McKenzie M, Bloch S, Moskowitz C, McKenzie DP, O'Neill I. Family focused grief therapy: a randomized, controlled trial in palliative care and bereavement. Am J Psychiatry 2006; 163:1208-18.

9. Kissane DW, Bloch S. Family grief. Br J Psychiatry 1994;164:72840. 